BV in Indian women. This study examined the risk factors for persistent bacterial vaginosis in young reproductive women in India. Study Design Between November 2005 and January 2007, a prospective cohort study was carried out to examine the relationship of BV and HSV-2 acquisition among women in Mysore. Quarterly, data were collected on sociodemographics, risk behaviour, partner characteristics, followed by a physical examination to diagnose and treat reproductive tract infections. BV was defined using Nugent scoring of gram stained vaginal smears. Persistent BV was calculated using generalised estimating equation methods. Women gave informed consent prior to enrolment in the study.

Results Of the 420 women for which there were data available for all three visits, 114 (27\%) had two or more BV episodes. Women with a history of 2 or more BV episodes were more likely to be infected with Trichomonas vaginalis [OR $72.93,95 \%$ CI 9.69 to 548.4 ] and be diagnosed with HSV-2 infection [OR 2.58, 95\% CI 1.44 to 4.63] compared to women with no BV history. Women with a history of BV were also more likely to report no education, tubal ligation, being a non-Muslim, and having a sex partner who had other sex partners.

Conclusions Young reproductive age women in India have a high persistence of BV. Although the association between BV and Trichomonas vaginalis is unclear, it seems prudent to recommend that women with BV or TV be screened for both infections.

\section{P3-S7.12 INFECTIONS AND INTERVENTIONS IN PREGNANT WOMEN AT HIGH RISK OF PRETERM BIRTH: A COHORT STUDY}

doi:10.1136/sextrans-2011-050108.495

1J Wilson, ${ }^{1}$ I Okpaluba, ${ }^{2} \mathrm{~N}$ Simpson. ' Leeds Teaching Hospitals NHS Trust, Leeds, UK; ${ }^{2}$ University of Leeds, Leeds, UK

Background Preterm birth (PTB) accounts for 65\% neonatal deaths and $50 \%$ neurological disability in children. Prior spontaneous PTB is the highest risk factor for subsequent PTB and is usually associated with sub-clinical infection, possibly due to genetic polymorphisms of proinflammatory cytokines. Bacterial vaginosis (BV) has been implicated in PTB and early treatment may reduce it but less attention has been paid to other infections. We assessed the infective and obstetric complications in a group of pregnant women at high risk of PTB and the interventions to reduce PTB.

Methods Study group: Pregnant women at high risk of PTB Interventions: Microbiological screening for infections from beginning of second trimester then 4-weekly until 28 weeks gestation with treatment of infections found; 2-weekly ultrasound assessment of cervical length during second trimester with cerclage and progesterone injections if needed. Outcome: Gestational age at delivery.

Results We have managed 104 pregnancies in 95 multiparous women who had at least one previous mid-trimester miscarriage (MTM), PTB or stillbirth due to chorioamnionitis. $21 \%$ had two previous MTM and/or PTB; $5 \%$ had three; $4 \%$ had four and $1 \%$ had five MTM/PTB. $75 \%$ were of white ethnicity, $7 \%$ asian, $18 \%$ black. One or more infection was identified in 51 (49\%) of the pregnancies; Group B streptococcal infection (GBS) in $21 \%$, BV in $16 \%$, S aureus in $3 \%$, heavy growth vaginal or urinary coliforms in $16 \%$. Prevalence of infections was more frequent in black (68\%), than white (48\%), than asian (14\%) women. Pregnancy outcome: Exact gestational age unknown in 4 due to transfer to different hospital when $>28 / 40$ gestation so outcomes for 100 pregnancies. Four resulted in MTM leaving 96 viable pregnancies. Gestational age at delivery: 24-27/40 in $3 \% ; 28-31 / 40$ in $3 \% ; 32-37 / 40$ in $13 \%$, term in $81 \%$. There was no association with treated infection and pregnancy outcome, infections had been identified and treated in 39\% of PTBs and $50 \%$ of term births.

Conclusions In this group of women with high risk pregnancies, $49 \%$ had infections known to be associated with PTB which were treated in the second trimester. The overall PTB rate was 19\% with $6 \%$ extremely or very preterm. There was no association between treated infection in pregnancy and PTB. Although the PTB rates are higher than the normal obstetric population they are significantly lower than would be predicted for such high risk pregnancies.

\section{P3-S7.13 EVALUATION OF THE AFFIRM VPIII MICROBIAL IDENTIFICATIONS TEST FOR THE DIAGNOSIS OF VAGINITIS AND BACTERIAL VAGINOSIS}

doi:10.1136/sextrans-2011-050108.496

${ }^{1} \mathrm{D}$ V Maseko, ${ }^{1} \mathrm{~F}$ M Radebe, ${ }^{1} \mathrm{~L}$ Gumede, ${ }^{1} \mathrm{~N}$ Bhojraj Sewphershad, ${ }^{1} \mathrm{~S}$ Khumalo, ${ }^{1}$ I Basson, ${ }^{1} \mathrm{D}$ Lewis. ${ }^{1}$ National Institute for Communicable Diseases (NHLS), Johannesburg, South Africa

Background Vaginitis and bacterial vaginosis (BV) are the most prevalent causes of vaginal symptoms among women of childbearing age. Diagnosis is based on clinical signs and symptoms, microscopy, $\mathrm{pH}$ and the whiff" test, and laboratory performed tests. The Affirm VPIII Microbial Identification test (Becton Dickinson) incorporates non-amplified DNA probes for Candida spp., Trichomonas vaginalis and Gardnerella vaginalis as an indicator for BV.

Objective To compare the Affirm VPIII Microbial Identification test for the detection of Candida spp., Gardnerella vaginalis and Trichomonas vaginalis to graded Gram stain for Bacterial Vaginosis and yeast as well as to an in-house Trichomonas vaginalis PCR (Polymerase Chain Reaction).

Methods In this study, specimens from 191 patients were evaluated. Specimens were collected consecutively from patients with vaginal discharge syndrome presenting at an STI (Sexually transmitted infection) clinic in Gauteng, South Africa between May 2010 and February 2011. Study inclusion was dependent upon the request and collection of both a vaginal swab for Affirm VP III and a second swab to make a smear for Gram staining. A cervical swab specimen was also collected for PCR at the same visit. Graded Gram stain for Bacterial vaginosis and yeast as well as an in-house real-time PCR method on the Rotorgene platform was performed in the laboratory. The sensitivity and specificity of the assay was determined using the graded Gram staining as a gold standard for BV and Candida spp. The real-time PCR was the gold standard for the Trichomonas vaginalis (TV).

Results Analysis of the Affirm VPIII gave a sensitivity of $98 \%$ for BV correctly categorising 80 of the $82 \mathrm{BV}$ positive specimens and specificity of $76 \%$ correctly identifying 38 of the 50 BV- negative specimens. The Affirm VPIII for Candida yielded a sensitivity of $86 \%$ and specificity of $95 \%$. When the Affirm was compared to the Real-time TV PCR the sensitivity was at $45 \%$ and specificity at $99 \%$. Conclusion The Affirm VPIII is an objective system which detects mixed vaginal infections and can be used in any setting. The performance characteristics of the Affirm VPIII for BV and Candida were comparable to those of other published studies. However in this study the Trichomonas vaginalis PCR was used as a gold standard therefore the sensitivity of the Affirm VPII of $45 \%$ is similar to that of culture or wet mount.

\section{P3-S7.14 THE ASSOCIATION OF UREAPLASMA UREALYTICUM WITH MALE NON-GONOCOCCAL URETHRITIS}

doi:10.1136/sextrans-2011-050108.497

${ }^{1} \mathrm{M}$ Frølund, ${ }^{2} \mathrm{P}$ Lidbrink, ${ }^{2} \mathrm{M}$ Cullberg, ${ }^{2} \mathrm{~A}$ Wikström, ${ }^{1} \mathrm{P}$ Ahrens, ${ }^{1} \mathrm{~J} \mathrm{~S}$ Jensen. ${ }^{1}$ Statens Serum Institute, Copenhagen, Denmark; ${ }^{2}$ Karolinska University Hospital, Sweden

Background The aetiology of non-gonococcal urethritis (NGU) is unexplained in $30-50 \%$ of cases. The role of ureaplasmas is not 
clear. We detected Ureaplasma urealyticum (Uu) and U parvum (Up) by quantitative PCR in the urine of men with and without NGU to show a possible association with urethritis.

Methods Urine samples from 158 male STD-clinic attendees with symptomatic NGU ( $>5 \mathrm{PMNL} / \mathrm{hpf}$ ) and 77 asymptomatic men without NGU ( $<5 \mathrm{PMNL} / \mathrm{hpf}$ ) were collected. The patient's age and number of partners within the previous 6 months were recorded. All samples were tested for Neisseria gonorrhoeae (Ng), Chlamydia trachomatis (Ct), Mycoplasma genitalium (Mg), Uu, Up, Trichomonas vaginalis (Tv), herpes simplex virus (HSV) 1 and 2, and adenovirus by real-time PCR.

Results Ct and Mg were found in 22 and $30 \%$ of NGU, respectively, and were associated with NGU ( $p<0.0001$ both). Three had dual Ct and $\mathrm{Mg}$ infection. Uu was detected in $13 \%$ of NGU cases and $12 \%$ of controls ( $p>0.99)$. The median Uu bacterial DNA load was higher in men with NGU than in men without (223 genome equivalents (geq) and 10 geq, respectively; $\mathrm{p}=0.002$ ). Using ROC-curve analysis to determine the optimal cut-off, patients with $>53$ geq were more likely to have urethritis $(p=0.02)$. In men with NGU of unknown aetiology, there was no difference in the rate of Uu detection when compared to controls $(p=0.26)$. The corresponding median $U u$ bacterial DNA load were significantly higher in this group than in controls $(p=0.01)$, and using a cut-off of $>53$ geq, men with NGU of unknown aetiology were more likely to harbour $U u(14 \%)$ than were men from the control group ( $1 \%),(p=0.005)$. Up was detected in $14 \%$ of NGU cases and $19 \%$ of controls $(p=0.34)$. There was no difference in the detection rate of Up or in the median Up bacterial load in any of the groups. HSV-1 was detected in $3 \%$ of cases and $1 \%$ of controls. HSV-2 was found in $2 \%$ of NGU cases. All urine samples were negative for adenovirus. Cases and controls had similar median number of partners within 6 months (2 partners) and age (28 and 29 years, respectively).

Conclusion The bacterial load of $U$ urealyticum in men with NGU and in men with NGU of unknown aetiology was higher than in men without NGU, and the presence of $>53$ geq of $U u$ was associated with urethritis in both groups. In accordance with other studies, U parvum was not associated with urethritis. (Preliminary results were presented at the ASM general meeting, San Diego 2010).

\section{P3-S7.15 DIAGNOSTIC RELIABILITY OF WET PREP MICROSCOPY FOR T VAGINALIS IN WOMEN VISITING A HIGH-VOLUME STD CLINIC}

doi:10.1136/sextrans-2011-050108.498

J Fitch, T Anderson, M Thrun, C Mettenbrink. Public Health Department, Denver, USA

Background For years, wet prep microscopy has been the cornerstone of testing for $T$ vaginalis due to the simplicity of the test and its low cost to perform. Though still recommended as a means of diagnosis for vaginal infections, its diagnostic sensitivity (typically $50 \%-70 \%$ ) falls short of other means of testing, including culture (approximately $80 \%$ sensitivity). This study compares the performance and costs of wet prep tests to culture in the hands of experienced laboratorians in a high volume STD clinic.

Methods The Denver Metro Health (STD) Clinic sees over 800 patients and performs over 100 wet prep tests every month. Between 1 January 2010 and 30 September 2010, a wet prep test and a $T$ vaginalis culture were performed on every female patient receiving a comprehensive exam, regardless of symptoms, as part of resistance testing for the STD Surveillance Network (SSuN). The results of the two tests were then compared.

Results Wet prep tests and $T$ vaginalis cultures were performed on 555 female patients. Of those, 51 were positive by wet prep and 62 were positive by culture see Abstract P3-S7.15 table 1. There were no wet prep false positives reported. The average material cost for each in-house wet prep was $\$ 0.32$ and the cost for each culture was $\$ 1.78$. In comparision, materials for PCR testing for $T$ vaginalis cost approximately $\$ 2$ per test. Staff time required to perform wet prep microscopy is less than either culture or PCR.

Conclusions The wet prep sensitivity rate of $82.2 \%$ when compared to culture (which, until recently has been the gold standard for trich testing) suggests that wet prep microscopy may be more useful than previously recognised in venues where clinicians and laboratorians gain experience by performing a large number of tests. Additionally, the costs, both in supply and staff expense, suggest that, in tight budgetary times, wet prep may continue to be a cornerstone of $T$ vaginalis testing, even with the availability of molecular amplification methods. These results, however, were limited to a single clinic during a single point in time. As next steps, wet prep testing might be compared to molecular amplification tests.

Abstract P3-S7.15 Table 1 Diagnostic reliability of wet prep microscopy for $T$ Vaginalis in women visiting a high-volume STD clinic

\begin{tabular}{lccc}
\hline & Wet prep & & \\
\cline { 2 - 3 } & Positive & Negative & Total \\
\hline Culture & & & \\
Positive & 51 & 11 & 62 \\
Negative & 0 & 493 & 493 \\
Total & 51 & 504 & 555 \\
\hline
\end{tabular}

\section{P3-S7.16 LACTOBACILLUS CRISPATUS COLONISATION REDUCES RISK OF BACTERIAL VAGINOSIS (BV) ACQUISITION}

doi:10.1136/sextrans-2011-050108.499

M Antonio, M Petrina, L Meyn, S Hillier. Magee-Womens Research Institute, University of Pittsburgh, Pittsburgh, USA

Background Longitudinal studies have found that vaginal colonisation by hydrogen peroxide-producing strains of lactobacilli is associated with a reduced risk of BV acquisition. Several Lactobacillus spp. including $L$ crispatus, $L$ jensenii, and $L$ gasseri can produce hydrogen peroxide, but no longitudinal studies to date have assessed whether all hydrogen peroxide producing species are equally protective. The objective of this study was to determine which species of Lactobacillus were associated with a reduced risk of BV acquisition.

Methods In this secondary analysis of women enrolled in a vaccine trial, 112 women aged 18-40 who had Gram stain Nugent scores $<6$ at study entry were followed bimonthly for up to 18 months for acquisition of BV. Vaginal and rectal swabs were collected at each visit for culture identification of lactobacilli. Species identification was performed by repetitive sequence PCR, and if necessary, 16S rRNA gene RFLP or sequencing. Cox proportional hazards models were used to assess the association between Lactobacillus colonisation at the prior visit and BV acquisition.

Results There were 40 BV acquisitions detected during 486 followup visits over 65.3 woman-years for an incidence of 61 per 100 woman-years. As shown in Abstract P3-S7.16 table 1, women colonised by $L$ crispatus were $53 \%$ less likely to acquire BV at a subsequent visit $(\mathrm{p}=0.023)$.

Conclusions $L$ crispatus was the only species protective against BV acquisition. Since neither $L$ jensenii nor $L$ gasseri reduced the risk of BV despite their capacity to produce hydrogen peroxide, other characteristics of $L$ crispatus may be more critical then peroxide production in protecting from BV acquisition. 